\title{
Effect of symbiotic supplementation on glycemic control, lipid profiles and microalbuminuria in patients with non- obese type 2 diabetes: a randomized, double-blind, clinical trial
}

Zarin sadat Ebrahimi ${ }^{1}$, Ensieh Nasli-Esfahani ${ }^{2}$, Azadeh Nadjarzade ${ }^{1,3}$ and Hassan Mozaffari-khosravi ${ }^{1,4^{*}}$

\begin{abstract}
Background: The prevalent raise of type 2 diabetes (T2D) around the globe, are creating higher risk for cardiovascular diseases (CVDs) and increasing strain on each country's health care budget in the world. Microalbuminuria has appeared as a key parameter in diabetic patients. Microalbuminuria is also related to increased cardiovascular morbidity in people who are non-obese diabetic. Some studies have suggested that consumption of symbiotic foods might help improve the metabolic profile, inflammatory factors and biomarkers of oxidative stress. The aim of trial was to determine the effect of symbiotic supplementation on glycemic control, lipid profiles and microalbuminuria in non-obese T2D.

Methods: In this randomized, double-blind, clinically controlled trial, 70 patients with T2D (28 females, 42 males) were randomly divided into two groups ( $n=35$ for each group). The symbiotic group (SG) consumed $500 \mathrm{mg} / \mathrm{d}$ of symbiotic supplementations containing probiotics (Lactobacillus family, Bifidobacterium family, Streptococus thermophilus), Prebiotics (Fructo oligosaccharide) and B group vitamins (1 mg), lactose (0.5 mg), malt-dextrin, magnesium saturate and the placebo group (PG) consumed capsules filled with row starch and also $B$ group vitamins (1 mg), lactose $(0.5 \mathrm{mg})$, malt-dextrin, magnesium saturate for 9 weeks. Fasting blood glucose (FBG), hemoglobin A1c ( $\mathrm{HbA1c})$, blood lipid profiles, 24-h dietary recalls, and anthropometric measurements were measured at the baseline and at the end of trial. SPSS software, version 16 was used to test the data and the results were expressed as mean \pm standard deviation. Paired samples T-Test were used to compare continuous variables within groups. Comparison between different groups was performed through two independent samples T-Test. In the absence of normal distribution, the comparison between the groups was made using non-parametric Wilcoxon on signed ranks and Mann-Whitney tests. $P$ values $<0.05$ was considered significant.

(Continued on next page)
\end{abstract}

\footnotetext{
* Correspondence: mozaffari.kh@gmail.com

'Department of Nutrition, Faculty of Health, Shahid Sadoughi University of

Medical Sciences, Bahonar Square, Central Building, Yazd, Iran

${ }^{4}$ Yazd Diabetic Research Center, Shahid Sadoughi University of Medical

Sciences, Yazd, Iran

Full list of author information is available at the end of the article
} 
(Continued from previous page)

Results: Symbiotic supplementation decreased significantly, FBG $(P=0.05)$ and HbA1c $(P<0.01)$. There were no significant differences in lipid profiles within and between the groups at the end of study $(P>0.05)$.

Microalbuminuria $(P<0.05)$ and HbA1c $(P<0.05)$ are increased significantly in $P G$ at the end of the study.

Furthermore, the mean changes of microalbuminuria and $\mathrm{HbA1c}$ experienced significant between the two groups.

There was significant reduction in urea between two groups from baseline $(P=0.051)$. No significant changes in baseline were shown in creatinine among the two groups or within either groups $(P>0.05)$.

Conclusion: The consumption of $500 \mathrm{mg} / \mathrm{d}$ symbiotic supplementation for 9 weeks could improve the HbA1c, BMl and Microalbuminuria in T2D. Although, No effect has been indicated on FBS, lipid profiles, urea and creatinine.

Trial Registration: The trial has been registered in the Iranian Registry of Clinical Trials IRCT2015072223284N1, identifier. Registered 21 May 2016 "retrospectively registered".

Keywords: Type 2 diabetes, Symbiotic, Probiotic, Microalbuminuria

\section{Background}

Type 2 diabetes (T2D) has been raised quickly in the world during the recent years [1]. Approximately 85$95 \%$ of patients with diabetic patients have T2D. It has been evaluated that $8 \%$ of adult in Iran are impressionable [1].

The prevalent raise of T2D around the world are developing higher risk for CVDs and raising species on health care budget of each country in the globe [2]. A specified category of risk factors associated to CVDs and T2D is frequently assigned to as the metabolic or the cardio metabolic disease. The diagnostic risk factors are basically consist hypertriglyceridemia, hyperglycemia, decreased high- to low-density-lipoprotein cholesterol ratio, insulin resistance and in certain descriptions microalbuminuria [3].

Secretion of urinary albumin above $20 \mu \mathrm{g} / \mathrm{min}$ (microalbuminuria) is forcefully prognostic of disease and death in patients who suffer from diabetes mellitus, especially T2D. Microalbuminuria has appeared as a key parameter in patients with T2 diabetes [4]. Also, the affiliation between albumin excretion and the risk of mortality is present, when other risk factors as sex, age, weight gain, smoking, ischemic heart disease and increased plasma lipid concentrations are derived from patients $[5,6]$. Also, it is a predictor of future infarcts, mortality and aftertime heart failure [7]. Because of the contrary effect of microalbuminuria on improvement in diabetic patients, and the renal risk of macroalbuminuria, testing and intervention plans must be performed primarily, at the microalbuminuria period [8].

Diabetic nephropathy is the usual reason for end stage renal disease happening [9]. In researches on patients with both Insulin Dependent Diabetes Mellitus (IDDM) and Non-Insulin Dependent Diabetes Mellitus (NIDDM), urinary secretion of albumin has been discovered to be predictor of improvement in proteinuria and end-stage DN [10].
In the last years, there has been raising portion in the microorganisms that inhibit the human micro biome [11]. Maximum of these micro biomes inhabit within our gastrointestinal tract, and their number and position are determined by host's phylogeny and diet [12]. It has been indicated that the gut microbiota combination is related to situations such as allergies, diabetes, cardiovascular disease, intestinal inflammatory disease, cancer and dyslipidaemia [13]. Variations in intestinal microbiota composed with specific nutrition can result in raised intestinal permeability leading via ultimate persistent condition of low-grade inflammation to the advancement of insulin resistance [14]. The function of probiotics in disease states has been studied largely, and the connections between various bacterial groups with insulin resistance, and diabetes are coming to clear [15]. Probiotics are beneficial because they have altered the mixture of the gut bacterial population and characterize the intake of nutrition and appetite [16], and also, they have illustrated useful effects in other diabetes-related situations containing body weight and inflammatory markers [17].

Alterations in the microflora may suppressed production of useful gut hormones such as glucagon-like peptide-1 (GLP-1), increased triglyceride production, insulin sensitivity, inflammation and changes in energy balance. Symbiotics are useful because they have changed the gut flora and increased GLP-1 and GLP-2 hormones. GLP-1 is promoting satiety and lowering glucose levels. Also, GLP-2 (glucagon like-peptide-2) is a proglucagon-derived peptide that decreases intestinal permeability. Secretion of GLP-1 and GLP-2 hormones may be associated with weight reduction, hypoglycemia and reduced HbA1c and lipid profiles [15].

Evidence of last research demonstrated a significant impact of gut microbiota on body's weight in patients with T2D. The consumption of probiotics has been shown to decrease inflammation and oxidative stress 
signs, and to recover glycemic and insulin metabolism in non-obese T2 diabetic patients [18].

Experimental data show that intestinal microbiota change with probiotics and prebiotics may positively affect the host's adiposity and glucose metabolism, but their effects are temporary and they would reduce gently after interruption. Today, it is usual to discover probiotic foods with added prebiotics, like a mixture, in which the concentration of prebiotic is typically below $10 \mathrm{~g} / \mathrm{kg}$ is known as a 'symbiotic'. In symbiotics existed the potential synergy between probiotics and prebiotics. Some studies have demonstrated that the usage of symbiotic foods might assist the management of the metabolic indexes, inflammatory agents and biomarkers of oxidative stress [19].

Lastly, A few publications have also explained that consumption of symbiotics may terminate modified insulin sensitivity and metabolic indexes in non-obese T2 diabetic patients [20]. The good affects of symbiotics on insulin metabolism and inflammatory markers could be interceded by the manufacture of short chain fatty acids, modulation of the intestinal microbiota composition, and reduced expression of inflammation-linked genes. Tajadadi et al. showed that the consumption of symbiotic food had advantageous effects on insulin metabolism among patients with diabetes [21].

The mixture of the probiotic and prebiotic foods might improve the living of the bacteria passing the superior section of the gastrointestinal tract, thereby elevating their beneficial effects in the colon [22]. The present study's purposes are to test the hypothesis that symbiotics can effect on glycemic control, lipid profiles, HbA1c and microalbuminuria in non-obese patients with T2D.

\section{Methods}

\section{Study design and participants}

This study was a single center, randomized, doubleblind, placebo-controlled trial. Eighty two patients with T2D (44 men and 38 women) were recruited from diabetic patients of Diabetes \& Metabolic Diseases Clinic 1 of Tehran University of Medical Sciences in Tehran, Iran. Recruitment was done by telephone and personally contact. All patients had been diagnosed with T2D for 5 years. T2D was diagnosed according to the criteria of world health organization (World Health Organization, 1985) [23]. These diabetic patients were 35 to 75 years old who were divided to symbiotic (SG) or placebo group (PG) subjects randomly and using a block randomization procedure with matched subjects in each block based on sex and age. The allocation of the SG or PG group was concealed from researchers and the symbiotic and placebo capsules had an identical appearance and packing. Therefore, neither the subjects nor the investigators were aware of the treatment assignments in this double-blinded study. Diabetic patients who have BMI under 35, have microalbuminuria and are consent to participate in the study, are not pregnant or in lactation were included in this study. Patients, who use antibiotic drugs or any new supplementation or drugs, the presence of kidney, liver, or inflammatory intestinal disease, thyroid disorders, immunodeficiency disease, required insulin injections, don't use symbiotics or placebos more than 10 consecutive days, migrate or die were excluded from this study and also, don't must be on angiotensin-converting enzyme inhibitor or angiotensin receptor blocker treatment. Each group consisted of 35 patients and they were followed up by telephone once a week.

The sample size was determined based on the primary information obtained from the study by Ejtehad et al. [24].The sample size was computed for $\alpha$ value equal to 0.05 and a power of $80 \%$. Patients in the SG received one symbiotic tablet daily that is receptacle $500 \mathrm{mg}$ probiotic and prebiotic and also, PG received one placebo tablet daily for 9 week. Patients were instructed to keep the symbiotic capsules in the refrigerator and were advised to keep their usual dietary habit, lifestyle, and physical activities and also, to avoid consuming any new drugs and supplementation without researcher's notice. Arrangement was made so that the patients would receive 15 days supply of their symbiotic capsules every 15 days. The symbiotic capsules contained Probiotics (Lactobacillus family, Bifidobacterium family, Streptococus thermophilus), Prebiotics (Fructo oligosaccharide) and $B$ group vitamins $(1 \mathrm{mg})$, lactose $(0.5 \mathrm{mg})$, maltdextrin, magnesium saturate and talc which were produced by Zist takhmir Company. The placebo capsules were filled from row starch and B group vitamins $(1 \mathrm{mg})$, lactose $(0.5 \mathrm{mg})$, malt-dextrin, magnesium saturate and talc. The placebo and symbiotic capsules are identical on similar flavor, odor and appearance.

\section{Measurements}

Information on food consumption, anthropometric measurements, and biochemical analyses (Fasting Blood glucose (FBG), Creatinine, Urea, Triglyceride (TG), Total Cholesterol (TC), HDL, LDL, HbA1c and urine sampling for determining $\mathrm{Alb} / \mathrm{Cr}$ ) were collected at the baseline and after intervention at the end of study. Nutrient intakes during 3 day were estimated using a 24-h food recall at the baseline and at the end of the trial. Threeday average were analyzed by Nutritionist IV software (N squared computing, San Bruno, Calif., USA). Anthropometric measurements were recorded by trained personnel. Body weight was measured using a scale (Seca, Hamburg, Germany) with $0.1 \mathrm{~kg}$ accuracy without shoes and with minimum clothing. Height was measured 
using a stadiometer (Seca) with $0.1 \mathrm{~cm}$ accuracy without shoes. BMI was calculated by dividing body weight (kilograms) by height (meters) squared. In the beginning and the end of study, $5 \mathrm{ml}$ blood and $5 \mathrm{ml}$ morning urine sample was drawn for each patient from the antecubital vein in the arm after 12 hour overnight fast.

FBG was measured using the standard enzymatic method with a Parsazmun kit (Karaj, Iran). Urea was measured using the photometric technique and parsazmun kit. Creatinine was measured by colorimetric Jaffe, kinetic. Glycated hemoglobin (HbA1c) was measured in the whole blood by HPLC method. TC and TG were measured by parsazmun kit and photometric method. Low density lipoprotein cholesterol (LDLc) and high density lipoprotein cholesterol (HDLc) were measured photometrical using parsazmun kit. For measuring random microalbuminuria using parsazmun kit and imunoturbidometric method. The total of biochemical analyses were done by equipped laboratory of Diabetes \& Metabolic Diseases Clinic 1 of Tehran University of Medical Sciences in Tehran.

\section{Data analysis}

SPSS software, version 16 was used to test the data and the results were expressed as mean \pm standard deviation. Paired samples T-Test were used to compare continuous variables within groups. Comparison between different groups was performed through two independent samples T-Test. In the absence of normal distribution, the comparison between the groups was made using non- parametric Wilcoxon on signed ranks and Mann-Whitney tests. $P$ values $<0.05$ was considered significant.

\section{Ethic consideration}

The present study was conducted according to the guidelines laid down in the Declaration of Helsinki and all procedures involving human persons were approved by the ethics committee at Yazd University of Medical Sciences and Health Services. Written informed consent was obtained from all patients. The trial has been registered in the Iranian Registry of Clinical Trials (http:// www.irct.ir, identifier: IRCT2015072223284N1 .

\section{Results}

According the Fig. 1; Six patients were excluded from the PG and also, 6 patients were excluded from the study in the SG. Eventually, this study was conducted on 70 patients, of which 28 were females and 42 males $(n=$ 35 for each group).According to Table 1, the patients' baseline characteristics did not differ significantly between the two groups $(P>0.05)$. According to our analysis, there was statistically significant increase in weight at the end of the trial in the PG $(P=0.005)$. BMI had significant differences at the end of study in PG and SG statistically $(P=0.012$ and $P=0.054$, respectively). The patients demonstrated good compliance with the capsule consumption and no adverse effects or symptoms were reported (Table 2).

Table 3 shows dietary intakes of patients throughout the study. As can see, there weren't any side effects

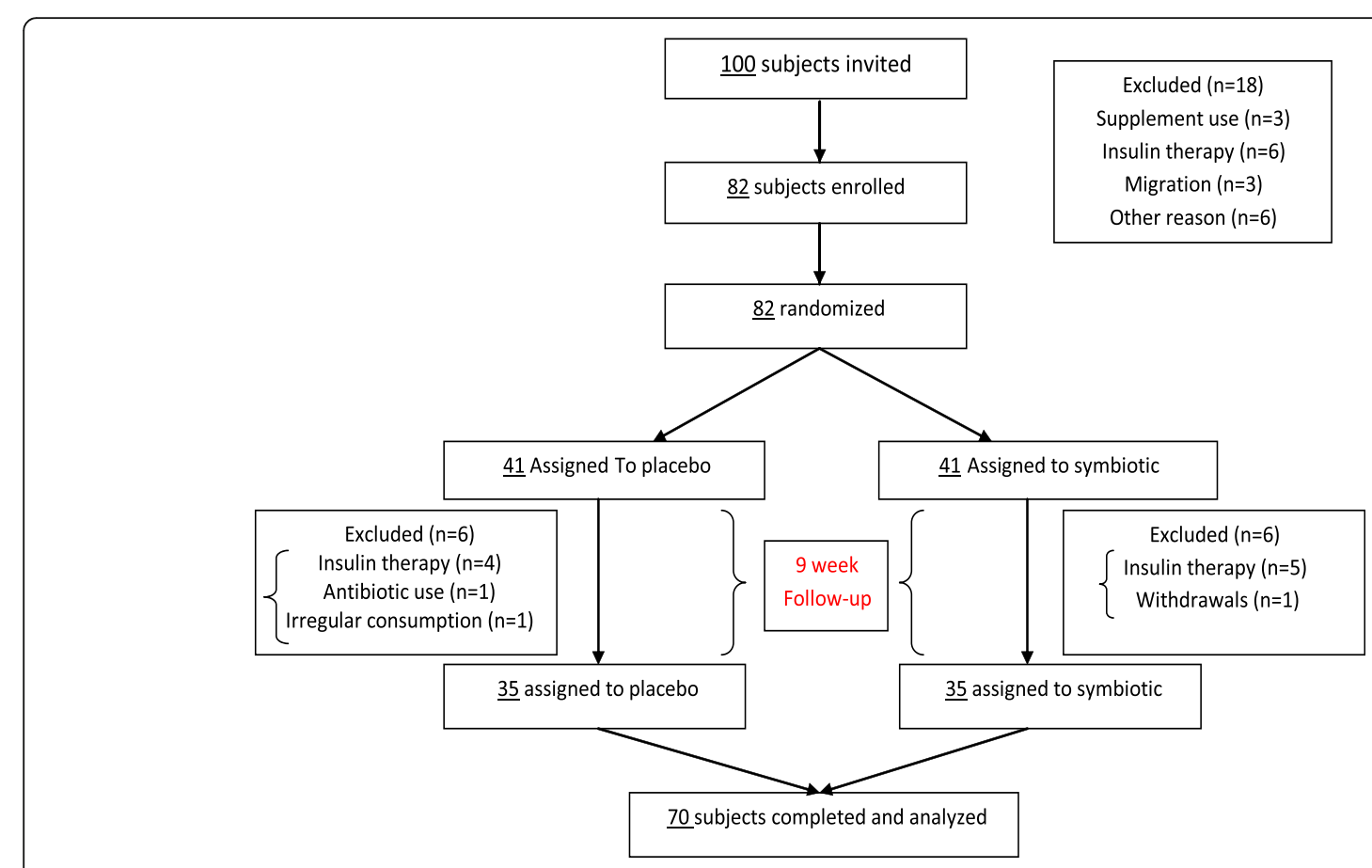

Fig. 1 Trial profile 
Table 1 Characteristics of study participants in baseline and after intervention

\begin{tabular}{llll}
\hline Variables & Symbiotic group $(n=35)$ & Placebo group $(n=35)$ & value $\rho$ \\
\hline Age (years) & $58.71 \pm 8.20$ & $58.63 \pm 8.06$ & 0.96 \\
Sex (male/female) & $(23 / 12)$ & $(19 / 16)$ & 0.09 \\
Height (cm) & $166.16 \pm 7.47$ & $165.44 \pm 9.71$ & 0.73 \\
Weight $(\mathrm{kg})$ Baseline & $77.59 \pm 10.54$ & $74.61 \pm 11.15$ & 0.25 \\
After & $77.50 \pm 10.54$ & $74.77 \pm 11.16$ & 0.29 \\
BMl $\left(\mathrm{kg} / \mathrm{m}^{2}\right)$ Baseline & $28.13 \pm 3.78$ & $27.30 \pm 3.81$ & 0.36 \\
After & $28.10 \pm 3.78$ & $27.38 \pm 3.81$ & 0.43 \\
Metformin (number/day) & $2.37 \pm 0.59$ & $2.40 \pm 0.55$ & 0.83 \\
Glibenclamide (number/day) & $1.03 \pm 0.74$ & $1.06 \pm 0.80$ & 0.87 \\
Glitazine (number/day) & $0.57 \pm 0.77$ & $0.60 \pm 0.73$ & 0.87 \\
Atrovastatine (number/day) & $0.89 \pm 0.71$ & $0.94 \pm 0.68$ & 0.73 \\
\hline
\end{tabular}

Data are presented as mean \pm Standard Deviation

$\mathrm{BMI}=$ Body Mass Index

${ }^{\mathrm{a}}$ Independent sample T-Test

reported following the consumption of symbiotic in patients within this study. The intake of monounsaturated fatty acid was significantly different between the SG and PG at baseline $(P=0.023)$ and the end of the study $(P=$ $0.023)$. No significant difference in energy and other nutrient intakes was observed between the two groups at the beginning and the end of the study $(P>0.05)$.

Table 4 demonstrates the effect of 9 weeks daily consumption of symbiotic and placebo on FBS, Urea, Creatinine, Lipid profiles, HbA1c and MI (urine Alb/Cr). According to Table 4, there was no statistically significant difference in Urea, Creatinine, Triglyceride, Cholesterol total, HDL-C and LDL-C between or within the groups at the end of the study $(P>0.05)$. There was no statistically significant difference in creatinine, HDL, LDL and HbA1c between the two groups at baseline of the study $(P>0.05)$. There was statistically significant difference in urea between the two groups at the baseline of the study $(P=0.051)$. Triglyceride and LDL-C were decreased in the SG compared with the PG, although the differences were not statistically significant $(P>0.05)$. FBG was significantly decreased in the SG $(P=0.050)$ and also, was decreased in the PG but not statistically significantly $(P=0.680)$. HDL-C was increased in the SG compared with the PG $(P=0.586$ and $P=0.287$ ), but wasn't statistically significantly.

HbA1c increased significantly from the baseline in the PG $(P=0.015)$ and decreased significantly at the end of

Table 2 Compare within two groups (Symbiotic \& Placebo groups)

\begin{tabular}{lcl}
\hline Variables & Symbiotic group $(n=35)$ & Placebo group $(n=35)$ \\
\hline Weight $(\mathrm{kg})$ value $^{\mathrm{a}} \rho$ & 0.07 & $0.001>$ \\
BMI $\left(\mathrm{kg} / \mathrm{m}^{2}\right)$ value $\rho$ & 0.05 & 0.01 \\
\hline
\end{tabular}

BMI = Body Mass Index

${ }^{\text {a Paired T-Test }}$ study in the SG $(P=0.000)$. As presented in Table 4 , microalbuminuria (MI) (Urine Alb/Cr) was different in the baseline and the complete the study in two groups.

Microalbuminuria (MI) in SG was decreased after intervention, but wasn't statistically significant $(P=$ $0.089)$, while $M I$ was significantly increased in the PG at the end of trial $(P=0.027)$.

\section{Discussion}

In the present study we observed significant effect of $500 \mathrm{mg}$ symbiotic supplementation on body weight, BMI, HbA1c and MI in diabetic patients ( 35 to $75 \mathrm{Y}$ ) for nine weeks. However, there was no effect on food intake except for MUFA. Million et al. in a meta-analysis showed that some Lactobacillus species were significantly affiliated with weight corrections in humans and animals: some Lactobacillus species were related to weight gain while other Lactobacillus species were related to weight loss or an anti-obesity effect [25]. Our study is favorable with Million et al. study [25].

Our results are compatible with the recent study by Sewify et al. in 2016 they evaluated the prevalence of urinary tract infection and antimicrobial susceptibility among diabetic patients with controlled and uncontrolled glycemia in Kuwait. They have provided a lot of evidence that the improvement of glycemia in diabetic patients might assist in decreasing the incidence of urinary tract infection in diabetic patients, chiefly in aged subjects [26]. In our study, we have also shown correlation between glycemic reduction and improvement of MI.

Our findings also have been demonstrated significant decrease in BMI in SG, and weight and BMI increased significantly in PG at the end of the study. In a Review of the studies that were done by Burcelin et al.it has been shown that the consumption of probiotics or 
Table 3 daily dietary intakes of patients throughout the study

\begin{tabular}{|c|c|c|c|}
\hline Variables & Before & After & value $^{a} \rho$ \\
\hline \multicolumn{4}{|l|}{ Energy (Kcal/day) } \\
\hline Placebo group & $1656.25 \pm 313.98$ & $1677.00 \pm 330.14$ & 0.40 \\
\hline Symbiotic group & $1746.31 \pm 430.06$ & $1742.34 \pm 372.62$ & 0.84 \\
\hline$\rho$ value & 0.32 & 0.44 & \\
\hline \multicolumn{4}{|l|}{ CHO (g/day) } \\
\hline Placebo group & $264.12 \pm 56.53$ & $268.32 \pm 55.81$ & 0.38 \\
\hline Symbiotic group & $277.94 \pm 79.45$ & $272.75 \pm 66.11$ & 0.37 \\
\hline$\rho$ value ${ }^{b}$ & 0.44 & 0.76 & \\
\hline \multicolumn{4}{|l|}{ Pro (g/day) } \\
\hline Placebo group & $84.25 \pm 117.69$ & $67.40 \pm 17.69$ & 0.39 \\
\hline Symbioti group & $68.43 \pm 17.53$ & $72.00 \pm 19.87$ & 0.12 \\
\hline$\rho$ value $e^{b}$ & 0.43 & 0.31 & \\
\hline \multicolumn{4}{|l|}{ Fat (g/day) } \\
\hline Placebo group & $41.55 \pm 15.82$ & $40.57 \pm 15.26$ & 0.71 \\
\hline Symbiotic group & $46.54 \pm 20.46$ & $46.26 \pm 18.49$ & 0.87 \\
\hline$\rho$ value $^{\mathrm{b}}$ & 0.25 & 0.16 & \\
\hline \multicolumn{4}{|l|}{ Cholesterol (g/day) } \\
\hline Placebo group & $157.45 \pm 153.76$ & $142.21 \pm 103.73$ & 0.57 \\
\hline Symbiotic group & $175.32 \pm 134.00$ & $168.67 \pm 117.51$ & 0.65 \\
\hline$\rho$ value $^{b}$ & 0.60 & 0.32 & \\
\hline \multicolumn{4}{|l|}{ SFA (g/day) } \\
\hline Placebo group & $12.65 \pm 3.83$ & $13.34 \pm 3.61$ & 0.22 \\
\hline Symbiotic group & $14.29 \pm 4.71$ & $15.01 \pm 4.58$ & 0.28 \\
\hline$\rho$ value $^{\mathrm{b}}$ & 0.11 & 0.09 & \\
\hline \multicolumn{4}{|l|}{ MUFA(g/day) } \\
\hline Placebo group & $11.27 \pm 4.26$ & $11.78 \pm 4.35$ & 0.39 \\
\hline Symbiotic group & $14.44 \pm 6.81$ & $14.70 \pm 6.00$ & 0.64 \\
\hline$\rho$ value $^{b}$ & 0.02 & 0.02 & \\
\hline \multicolumn{4}{|l|}{ PUFA (g/day) } \\
\hline Placebo & $6.15 \pm 3.88$ & $6.72 \pm 4.35$ & 0.21 \\
\hline Symbiotic Supplement & $9.45 \pm 10.89$ & $8.99 \pm 9.83$ & 0.18 \\
\hline$\rho$ value $^{b}$ & 0.09 & 0.21 & \\
\hline \multicolumn{4}{|l|}{ Fiber (g/day) } \\
\hline Placebo group & $12.77 \pm 6.00$ & $14.97 \pm 14.17$ & 0.38 \\
\hline Symbiotic group & $12.07 \pm 6.31$ & $11.83 \pm 5.31$ & 0.73 \\
\hline$\rho$ value $^{b}$ & 0.63 & 0.22 & \\
\hline \multicolumn{4}{|l|}{ Vitamin E (g/day) } \\
\hline Placebo group & $6.92 \pm 13.17$ & $3.00 \pm 2.72$ & 0.09 \\
\hline Symbiotic group & $4.38 \pm 4.72$ & $4.04 \pm 4.33$ & 0.32 \\
\hline$\rho$ value $^{\mathrm{b}}$ & 0.28 & 0.23 & \\
\hline \multicolumn{4}{|l|}{ Vitamin C (g/day) } \\
\hline Placebo group & $56.13 \pm 30.82$ & $60.46 \pm 33.27$ & 0.24 \\
\hline Symbiotic group & $51.58 \pm 34.91$ & $56.83 \pm 40.29$ & 0.32 \\
\hline
\end{tabular}


Table 3 daily dietary intakes of patients throughout the study (Continued)

\begin{tabular}{|c|c|c|c|}
\hline$\rho$ value $^{b}$ & 0.56 & 0.68 & \\
\hline \multicolumn{4}{|l|}{ Selenium (g/day) } \\
\hline Placebo group & $0.02 \pm 0.02$ & $0.02 \pm 0.01$ & 0.76 \\
\hline Symbiotic group & $0.02 \pm 0.01$ & $0.03 \pm 0.01$ & 0.10 \\
\hline$\rho$ value $^{\mathrm{b}}$ & 0.82 & 0.30 & \\
\hline \multicolumn{4}{|l|}{ Sodium(g/day) } \\
\hline Placebo group & $1982.19 \pm 1127.23$ & $2177.36 \pm 1492.55$ & 0.35 \\
\hline Symbiotic group & $2374.75 \pm 1328.88$ & $2166.70 \pm 866.06$ & 0.41 \\
\hline$\rho$ value $^{\mathrm{b}}$ & 0.18 & 0.97 & \\
\hline \multicolumn{4}{|l|}{ Potassium (g/day) } \\
\hline Placebo group & $1333.90 \pm 411.98$ & $1354.86 \pm 379.64$ & 0.70 \\
\hline Symbiotic group & $1427.55 \pm 443.20$ & $1385.84 \pm 306.36$ & 0.47 \\
\hline$\rho$ value ${ }^{b}$ & 0.36 & 0.70 & \\
\hline
\end{tabular}

Data are presented as mean \pm Standard Deviation

b: Student t-test; ${ }^{\text {a }}$ Paired T-Test

CHO: carbohydrate Pro: protein SFA: saturated fatty acid MUFA: mono unsaturated fatty acid PUFA: poly unsaturated fatty acid

symbiotics on diabetic patients might be the effect of glycemia decrease, body weight and BMI [12]. These findings are supported our result. Our findings also, showed consumption of symbiotics supplementation for 9 weeks reduced body weight and BMI in patients with T2D significantly.

Recently, a randomized, double-blind, placebocontrolled study was conducted on 20 volunteers (10 placebo group and 10 symbiotic group), aged 50-60 years for 30 days. Their results of the symbiotic group showed a significant increase $(p<0.05)$ in HDL cholesterol, non-significant reduction $(\mathrm{p}>0.05)$ in total cholesterol and triglycerides and a significant reduction $(p<$ $0.05)$ in FBG and no significant changes were reported in the placebo group [22]. In our study only FBG was significantly decreased in the SG $(P=0.050)$, but there were non-significantly decrease in triglycerides $(p=$ $0.082)$, TC $(P=0.805)$, LDLc $(P=0.786)$ and nonsignificantly increase in HDLc $(P=0.586)$.

In a systematic review of the clinical evidence that was done by Ruan et al. It hase been shown that probiotic consumption can control glycemic moderately and better. Also, they were expressed that modification of intestinal microbiota by probiotic supplementation can be a procedure for preventing hyperglycemia in clinical action [27]. This study is correspondent with our results. Hence, more research on effect of symbiotic are needed to do in future studies.

There are publications that show probiotics lower LDL cholesterol, however the data is conflicting [28, 29]. Agerholm-Larsen et al. reported that the consumption of yogurt fermented with different probiotics had uncertain effect on LDLc in obese subjects, they also expressed that hypolipidemic effect is dependent on the species of probiotics [30]. Accordingly, in our results there were no significant changes in LDLc in SG and PG at the end of the trial.

Previous studies regarding the antidiabetic properties of symbiotics has been limited to studies about the effects of symbiotics on FBG, insulin resistance or antioxidant status. Tajadadi-Ebrahimi et al. showed that the consumption of symbiotic bread for 8 weeks had advantageous effects on insulin metabolism among patients with diabetes [21]. In another double-blind, clinical trial that was done by Akram Kooshki et al. about the effect of symbiotics on inflammatory markers on patients with T2DM, has expressed consumption of symbiotic once a day for 8 weeks may decreased serum hs-CRP, IL-6 and TNF- $\alpha$ concentration, which are risk factors for CVD diseases [31].

In this study, we investigated effect of symbiotics not only on FBS, triglyceride, cholesterol, LDL, HDL and HbA1c, but also investigated on MI.

The pilot scale trial about the effect of probiotic dietary supplementation in patients with stage 3 and 4 chronic kidney disease has been done in 2009 by Ranganathan et al. in Canada which was performed on 13 subjects, 40 to 70 years old, with chronic kidney disease. Their results indicated that Mean biochemical group (Complete blood counts, serum biochemical testing, creatinine and urine protein) values for all patients either reduced or remained fixed during probiotic bacterial supplementation, but either remained comparatively fixed or significantly raised during placebo supplementation [32], In our results, only urine protein reduction is compatible with Ranganathan et al.'s results, that proteinuria in our study specified by microalbuminuria. Their mechanisms are perhaps suggestive by 
Table 4 Comparison mean of FBS, Urea, Creatinine, Lipid profiles, HbA1c and Ml in two groups

\begin{tabular}{|c|c|c|c|c|}
\hline Variables & Before & After & value $^{a} \rho$ & changes \\
\hline \multicolumn{5}{|l|}{$\mathrm{FBG}(\mathrm{mg} / \mathrm{dl})$} \\
\hline Placebo group & $132.74 \pm 32.45$ & $130.23 \pm 29.58$ & 0.68 & $2.51 \pm 35.97$ \\
\hline Symbiotic group & $142.34 \pm 40.59$ & $132.11 \pm 37.85$ & 0.05 & $10.22 \pm 29.79$ \\
\hline$\rho$ value ${ }^{b}$ & 0.27 & 0.81 & & 0.33 \\
\hline \multicolumn{5}{|l|}{ Urea (mg/dl) } \\
\hline Placebo group & $36.80 \pm 14.79$ & $37.94 \pm 14.57$ & 0.36 & $1.14 \pm 7.30-$ \\
\hline Symbiotic group & $31.20 \pm 7.67$ & $33.25 \pm 7.61$ & 0.10 & $2.05 \pm 7.31-$ \\
\hline$\rho$ value ${ }^{b}$ & 0.05 & 0.09 & & 0.60 \\
\hline \multicolumn{5}{|l|}{ Creatinine (mg/dl) } \\
\hline Placebo group & $1.05 \pm 0.22$ & $1.03 \pm 0.24$ & 0.22 & $0.02 \pm 0.11$ \\
\hline Symbiotic group & $1.04 \pm 0.26$ & $1.05 \pm 0.26$ & 0.82 & $-0.00 \pm 0.09$ \\
\hline$\rho$ value $^{b}$ & 0.91 & 0.73 & & 0.73 \\
\hline \multicolumn{5}{|l|}{ Triglycerides (mg/dl) } \\
\hline Placebo group & $122.00 \pm 46.14$ & $126.51 \pm 60.86$ & 0.62 & $4.51 \pm 54.51-$ \\
\hline Symbiotic group & $144.94 \pm 78.54$ & $130.37 \pm 64.59$ & 0.08 & $14.57 \pm 48.02$ \\
\hline$\rho$ value ${ }^{b}$ & 0.14 & 0.79 & & 0.12 \\
\hline \multicolumn{5}{|l|}{ Cholesterol (mg/dl) } \\
\hline Placebo group & $146.09 \pm 28.26$ & $149.09 \pm 40.82$ & 0.54 & $3.0 \pm 28.63-$ \\
\hline Symbiotic group & $145.86 \pm 30.50$ & $144.74 \pm 31.16$ & 0.80 & $1.11 \pm 26.47$ \\
\hline$\rho$ value & 0.97 & 0.61 & & 0.53 \\
\hline \multicolumn{5}{|l|}{$\mathrm{HDL}-\mathrm{C}(\mathrm{mg} / \mathrm{dl})$} \\
\hline Placebo group & $46.74 \pm 9.86$ & $45.62 \pm 12.49$ & 0.28 & $1.11 \pm 6.09$ \\
\hline Symbiotic group & $45.65 \pm 12.64$ & $46.20 \pm 11.00$ & 0.58 & $0.54 \pm 5.84-$ \\
\hline$\rho$ value ${ }^{b}$ & 0.69 & 0.84 & & 0.25 \\
\hline \multicolumn{5}{|l|}{ LDL-C (mg/dl) } \\
\hline Placebo group & $77.74 \pm 18.51$ & $78.80 \pm 24.63$ & 0.76 & $1.05 \pm 21.09-$ \\
\hline Symbiotic group & $77.20 \pm 18.74$ & $76.37 \pm 17.79$ & 0.78 & $0.83 \pm 17.93$ \\
\hline$\rho$ value $^{b}$ & 0.90 & 0.63 & & 0.68 \\
\hline \multicolumn{5}{|l|}{$\mathrm{HbA1C}(\%)$} \\
\hline Placebo group & $7.50 \pm 0.87$ & $7.77 \pm 1.13$ & 0.01 & $-0.26 \pm 0.60$ \\
\hline Symbiotic group & $7.44 \pm 0.98$ & $7.13 \pm 0.89$ & 0.00 & $0.30 \pm 0.46$ \\
\hline$\rho$ value $e^{b}$ & 0.76 & 0.01 & & $0.001>$ \\
\hline \multicolumn{5}{|l|}{ Urine Alb/Cr (mg/gr) } \\
\hline Placebo group & $62.77 \pm 59.69$ & $81.09 \pm 81.58$ & 0.02 & $-18.31 \pm 46.78$ \\
\hline Symbiotic group & $45.39 \pm 38.85$ & $34.94 \pm 13.11$ & 0.08 & $10.44 \pm 35.26$ \\
\hline$\rho$ value $e^{\mathrm{b}}$ & 0.15 & 0.00 & & $0.001>$ \\
\hline
\end{tabular}

Data are presented as mean \pm Standard Deviation

${ }^{\text {a }}$ Paired t-test

${ }^{\mathrm{b}}$ Student t-test

FBG: fasting blood glucose LDL-C: low density lipoprotein cholesterol HDL-C: high density lipoprotein cholesterol Urine Alb/Cr: urine

albumin/creatin Microalbuminuria

dihydropyridine calcium channel blockers which are not as impressive as ACE (Angiotensin-Converting-Enzyme) inhibitors or angiotensin receptor blocker (ARB) in reducing albuminuria [8]. High glucose products abnormally high amounts of free radicals by autoxidation of glucose and protein glycation, and oxidative stress has been reported to be a causal agent of tubule interstitial fibrosis in patients with diabetic nephropathy (DN) [33]. In fact, mechanisms of probiotics or symbiotics are challenging with pathogenic microorganisms for intestinal 
epithelial receptors, which leave useful antimicrobial combinations that activates pathogenic microbes and alter and block toxins [15].

Consonant to our study, several previous studies in humans and animals have suggested that nutrition of probiotic supplemented milk product (dahi/yoghurt) prevented development of diet induced T2D and metabolic disturbances [34, 35]. These studies also indicated that the consumption of probiotic formulations excluded obesity and diabetes in high fat diet fed to mice, and decreased food intake dramatically [2].

In our searches, there was not any publication about effect of probiotics or symbiotics on MI in patients with T2D found. Therefore, we think this study may to be appendage the beginning of studies about the effect of symbiotics on DN.

The present study, indeed, has reported the first evidence of improved microalbuminuria in diabetic patients. This result is by significant decrease in HbAlc and MI between two groups $(P=0.011$ and $P=0.002$, respectively). The exact mechanisms involved in the anti diabetic effects of symbiotics has remained unknown. These effects are perhaps partly related to symbiotic effect on gastrointestinal digestion and absorption.

The limitation of this study included its small sample size, short duration of study. Thus, further studies with bigger sample size are needed to confirm the positive effect of symbiotic on management of diabetes, particularly on MI in diabetic patients.

\section{Conclusion}

The consumption of $500 \mathrm{mg} / \mathrm{d}$ symbiotic supplementation for 9 weeks, decreased significantly fasting blood glucose, HbA1c, BMI and microalbuminuria in T2D patients. However, there were no significant changes in lipid profiles, urea and creatinine at the end of the study.

\section{Abbreviations \\ Alb/Cr: Albumin/creatinin ratio; BMl: Body mass index; DN: Diabetic Nephropathy; FBG: Fasting Blood Glucose; HbA1c: Hemoglobin A1c; HDLc: High Density Lipoprotein cholesterol; hs -CRP: High-sensitivity C-reactive protein; IDDM: Insulin Dependent Diabetes Mellitus; LDLc: Low Density Lipoprotein cholesterol; MI: Microalbuminuria; NIDDM: Non-Insulin Dependent Diabetes Mellitus; PG: Placebo Group; SG: Symbiotic Group; T2D: Type 2 diabetes; TC: Total Cholesterol; TG: Triglyceride; TNF a: Tumor Necrosis Factor a}

\section{Acknowledgements}

This study was supported by Yazd University of Medical Sciences and Health Services of Yazd of Iran. The authors thank the staff of Diabetes \& Metabolic Diseases Clinic 1 of Tehran University of Medical Sciences in Tehran, Iran for valuable assistance the authors also gratefully acknowledge the cooperation of the participating patients. Also, the authors thank Dr MH Lotfi for helping to compute sample size of trial.

\section{Funding}

No external funding for this study was used. The Yazd University of Medical Sciences and Health services covered some costs associated with the development and the publishing of the present manuscript. The corresponding author had full access to the data and final responsibility for the submission of the publication.
No writing assistance was utilized in the production of this manuscript

\section{Availability of data and materials}

Data available on request from the authors. The data that supports the findings of this study is available from the corresponding author upon reasonable request.

\section{Authors' Contributions}

ZSE performed searches of studies, recruited the volunteer patients with diabetes and filled consent with cooperation of patients and inscribed the manuscript. HM-K accomplished statistically analysis, modified this article and participated in the design of the study. EN-E participated in the design of the study and coordination to recruit the diabetic patients in laboratory. AN participated in its design and helped draft the manuscript. All authors read and approved the final manuscript.

\section{Competing interest}

The authors declare that they have no competing interests.

\section{Consent for publication}

In this study, there are no images or details on individuals reported within the manuscript, so according to (http://www.biomedcentral.com/ submissions/editorial-policies\#consent+for+publication) consent for publication of images may not be required. Written informed consent about usage of biochemical data was obtained from all patients.

\section{Ethics Approval and Consent to Participate}

The present study was conducted according to the guidelines laid down in the Declaration of Helsinki and all procedures involving humans were approved by the ethics committee at Yazd University of Medical Sciences and Health Services and the reference number is/17/1/227678. Written informed consent was obtained from all patients.

\section{Publisher's Note}

Springer Nature remains neutral with regard to jurisdictional claims in published maps and institutional affiliations.

\section{Author details}

${ }^{1}$ Department of Nutrition, Faculty of Health, Shahid Sadoughi University of Medical Sciences, Bahonar Square, Central Building, Yazd, Iran.

${ }^{2}$ Endocrinology and Metabolism Researcher Center, Endocrinology and Metabolism Clinical Sciences Institute, Tehran University of Medical Sciences, Tehran, Iran. ${ }^{3}$ Nutrition and Food Security Research Centre, School of Public Health, Shahid Sadoughi University of Medical Sciences, Yazd, Iran. ${ }^{4}$ Yazd Diabetic Research Center, Shahid Sadoughi University of Medical Sciences, Yazd, Iran

Received: 14 January 2017 Accepted: 23 May 2017

Published online: 02 June 2017

\section{References}

1. Peykari $\mathrm{N}$, et al. Diabetes research in Middle East countries; a scientometrics study from 1990 to 2012. J ResMed Sci. 2015;20(3):253.

2. Yadav H, Shalini J, Francesco M. Probiotics mediated modulation of gut flora might be biotherapeutical approach obesity and type 2 diabetes. Metabolomics 2011:1(3)

3. Stenman L, Burcelin R, Lahtinen S. Establishing a causal link between gut microbes, body weight gain and glucose metabolism in humans-towards treatment with probiotics. Benefic Microbes. 2016;7(1):11-22.

4. Schmitz A. Microalbuminuria, Blood Pressure, Metabolic Control, and Renal Involvement: Longitudinal Studies in White Non-Insulin-Dependent Diabetic Patients. Am J Hypertens. 1997;10(9):189S-97S.

5. Damsgaard EM, et al. Microalbuminuria as predictor of increased mortality in elderly people. BMJ. 1990;300(6720):297-300.

6. Allawi J, et al. Microalbuminuria in non-insulin-dependent diabetes: its prevalence in Indian compared with Europid patients. Br Med J (Clin Res Ed). 1988;296(6620):462-4.

7. Gerstein $\mathrm{HC}$, et al. Albuminuria and risk of cardiovascular events, death, and heart failure in diabetic and nondiabetic individuals. JAMA. 2001;286(4):421-6.

8. Wu A, et al. An alarmingly high prevalence of diabetic nephropathy in Asian type 2 diabetic patients: the MicroAlbuminuria Prevalence (MAP) Study. Diabetologia. 2005;48(1):17-26. 
9. Tavafi M. Diabetic nephropathy and antioxidants. J Nephropathol. 2013;2(1):20-7.

10. Klein R, Klein BE, Moss SE. Prevalence of microalbuminuria in older-onset diabetes. Diabetes Care. 1993;16(10):1325-30.

11. Lambeth SM, et al. Composition, Diversity and Abundance of Gut Microbiome in Prediabetes and Type 2 Diabetes. J diabetes obes. 2015;2(3):1.

12. Burcelin R, et al. Gut microbiota and diabetes: from pathogenesis to therapeutic perspective. Acta Diabetol. 2011;48(4):257-73.

13. Gomes AC, et al. Gut microbiota, probiotics and diabetes. Nutr J. 2014;13(1):1.

14. Bekkering $P$, et al. The intricate association between gut microbiota and development of type 1, type 2 and type 3 diabetes. Expert Rev Clin Immunol. 2013;9(11):1031-41.

15. Shane-McWhorter L. Dietary supplements and probiotics for diabetes. AJN Am J Nurs. 2012;112(7):47-53.

16. Hariri $\mathrm{M}$, et al. The effect of probiotic soy milk and soy milk on anthropometric measures and blood pressure in patients with type II diabetes mellitus: A randomized double-blind clinical trial. ARYA atherosclerosis. 2015;11(Suppl 1):74.

17. Firouzi S. et al. Effect of multi-strain probiotics (multi-strain microbial cell preparation) on glycemic control and other diabetes-related outcomes in people with type 2 diabetes: a randomized controlled trial. Eur J Nutr. 2016: p. 1-16. doi:10.1007/s00394-016-1199-8.

18. Fallucca $F$, et al. Influence of diet on gut microbiota, inflammation and type 2 diabetes mellitus. First experience with macrobiotic Ma-Pi 2 diet. Diabetes Metab Res Rev. 2014;30(S1):48-54.

19. Asemi Z, et al. Effects of synbiotic food consumption on metabolic status of diabetic patients: a double-blind randomized cross-over controlled clinical trial. Clin Nutr. 2014;33(2):198-203.

20. Shakeri $\mathrm{H}$, et al. Consumption of synbiotic bread decreases triacylglycerol and VLDL levels while increasing HDL levels in serum from patients with type-2 diabetes. Lipids. 2014;49(7):695-701.

21. Tajadadi-Ebrahimi M, et al. Effects of daily consumption of synbiotic bread on insulin metabolism and serum high-sensitivity C-reactive protein among diabetic patients: a double-blind, randomized, controlled clinical trial. Ann Nutr Metab. 2014;65(1):34-41.

22. Moroti $\mathrm{C}$, et al. Effect of the consumption of a new symbiotic shake on glycemia and cholesterol levels in elderly people with type 2 diabetes mellitus. Lipids Health Dis. 2012;11(1):1.

23. Organization WH. Diabetes Mellitus: Report of a WHO Study Group [meeting held in Geneva from 11 to 16 February 1985]. 1985.

24. Ejtahed $\mathrm{HS}$, et al. Probiotic yogurt improves antioxidant status in type 2 diabetic patients. Nutrition. 2012;28(5):539-43.

25. Million $\mathrm{M}$, et al. Comparative meta-analysis of the effect of Lactobacillus species on weight gain in humans and animals. Microb Pathog. 2012;53(2):100-8.

26. Sewify $\mathrm{M}$, et al. Prevalence of Urinary Tract Infection and Antimicrobial Susceptibility among Diabetic Patients with Controlled and Uncontrolled Glycemia in Kuwait. J diabetes res. 2015;2016.

27. Ruan $Y$, et al. Effect of probiotics on glycemic control: a systematic review and meta-analysis of randomized, controlled trials. PLoS One. 2015;10(7): e0132121.

28. Xiao JZ, et al. Effects of milk products fermented by Bifidobacterium longum on blood lipids in rats and healthy adult male volunteers. J Dairy Sci. 2003;86(7):2452-61.

29. Taylor G, Williams CM. Effects of probiotics and prebiotics on blood lipids. Br J Nutr. 1998;80(4):S225-30

30. Agerholm-Larsen $L$, et al. Effect of 8 week intake of probiotic milk products on risk factors for cardiovascular diseases. Eur J Clin Nutr. 2000;54(4):288-97.

31. Kooshki AA, Tofighiyan T, Rakhshani MH. Effects of synbiotics on inflammatory markers in patients with type 2 diabetes mellitus. Global $J$ health sci. 2015;7(7):1.

32. Ranganathan $\mathrm{N}$, et al. Probiotic dietary supplementation in patients with stage 3 and 4 chronic kidney disease: a 6-month pilot scale trial in Canada. Curr Med Res Opin. 2009;25(8):1919-30.

33. Lu Y-C, et al. Effect of Lactobacillus reuteri GMNL-263 treatment on renal fibrosis in diabetic rats. J Biosci Bioeng. 2010;110(6):709-15.

34. Yadav H, Jain S, Sinha P. Antidiabetic effect of probiotic dahi containing Lactobacillus acidophilus and Lactobacillus casei in high fructose fed rats. Nutrition. 2007;23(1):62-8.

35. Yadav $\mathrm{H}$, Jain $\mathrm{S}$, Sinha PR. Oral administration of dahi containing probiotic Lactobacillus acidophilus and Lactobacillus casei delayed the progression of streptozotocin-induced diabetes in rats. J Dairy Res. 2008;75(02):189-95.

\section{Submit your next manuscript to BioMed Central and we will help you at every step:}

- We accept pre-submission inquiries

- Our selector tool helps you to find the most relevant journal

- We provide round the clock customer support

- Convenient online submission

- Thorough peer review

- Inclusion in PubMed and all major indexing services

- Maximum visibility for your research

Submit your manuscript at www.biomedcentral.com/submit
Biomed Central 